
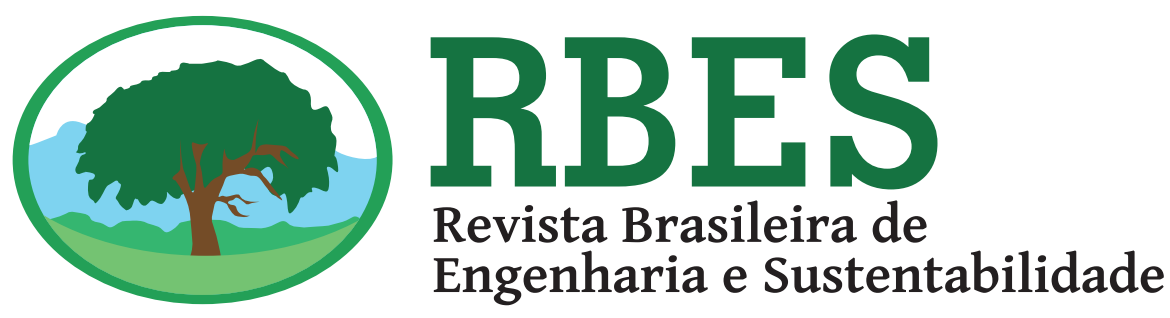

\title{
ANÁLISE DA PERDA DE CARGA EM COLETORES SOLARES DE BAIXO CUSTO QUANTO ÀS CARACTERÍSTICAS CONSTRUTIVAS
}

CORREA, I. B. '; SÁ, J. S. ${ }^{2}$

1 Tecnológo em Saneamento Ambiental - Instituto Federal Sul-Rio-Grandense - Campus Pelotas.

2 Prof. Dr., Instituto Federal Sul-Rio-Grandense - Campus Pelotas.

Palavras-chave:

Solar, Energia, sustentabilidade, eficiência.

\section{Resumo}

A energia solar é uma forma efetiva e sustentável para a redução do consumo de energia elétrica. Os sistemas de aquecimento solar da água podem operar com fluxo natural ou forçado, dependendo do porte da instalação. O objetivo desse estudo foi quantificar as perdas de carga em coletores solares de baixo custo em relação às características do condutor do fluido, ao sentido do fluxo do fluido no interior do coletor e ao arranjo de montagem dos coletores, quando submetidos à variação de vazão. 0 coletor $\mathrm{C} 1$ foi constituído por tubos de Policloreto de Vinila (PVC) de $20 \mathrm{~mm}$ de diâmetro, garrafas de Politereftalato de Etileno (PET) e embalagens longa vida. 0 coletor $\mathrm{C} 2$ foi montado em forros de PVC com as dimensões de 0,2 m de largura e 0,08 m de espessura e o coletor C3 foi constituído por forros de PVC com 0,14 m de largura e 0,014 m de espessura. As dimensões dos condutos dos coletores e a vazão do fluido influenciam significativamente na perda de carga dos coletores. 0 layout construtivo que apresentou resultados positivos foi o coletor constituído de tubos de PVC, instalado em arranjo paralelo, com fluxo de água no sentido paralelo e vazão inferior a 1,0 $\mathrm{m}^{3} \mathrm{~h}^{-1}$. Palavras-chave: Aquecimento Solar, Energia, sustentabilidade, eficiência.

\section{ANALYSIS OF THE HEAD LOSS IN SOLAR COLLECTORS OF LOW COST AS TO THE CONSTRUCTIVE CHARACTERISTICS.}

Keywords: Solar heating,

Energy, sustainability, efficiency.

\begin{abstract}
Solar energy is an effective and sustainable way to reduce electricity consumption. Solar water heating systems can operate with natural or forced flow, depending on the size of the facility. The objective of this study was to quantify the load losses in three models of low cost collectors (C1, C2 and C3) when subjected to flow variations in forced flow regime in relation to the material used in the collection of the collectors, to the flow direction of the fluid and the installation arrangement of the collectors. The C1 collector consisted of Polyvinyl Chloride (PVC) pipes with $20 \mathrm{~mm}$ diameter, Polyethylene terephthalate (PET) bottles and long life packages. The collector C2 was assembled with PVC lining with the dimensions of $0.2 \mathrm{~m}$ wide and $0.08 \mathrm{~m}$ thick and the collector C3 was constituted with $0.14 \mathrm{~m}$ of width and $0.014 \mathrm{~m}$ of thickness. The dimensions of the collector conduits and the fluid flow influence the load loss of the collectors. The constructive layout that presented positive results was the PVC pipe collector, installed in a parallel arrangement, with water flow in the parallel direction and flow rate less than $1 \mathrm{~m}^{3} \mathrm{~h}^{-1}$.
\end{abstract}




\section{INTRODUÇÃO}

A energia solar é uma fonte de energia renovável e é considerada soluçáo ideal para o abastecimento energético de áreas remotas e para a redução do uso de fontes de energia fósseis. Um país como o Brasil tem potencialidades para desenvolver a indústria da energia solar e ampliar a participação dessa energia na matriz energética (SILVA e CARMO, 2017; GOMES, 2012). A utilização da energia solar em sistemas de aquecimento de água residencial significa uma economia de energia elétrica para o consumidor final e um incentivo ao uso de energias provenientes de fontes renováveis (LOPO, 2010).

Em razão desse fato, o aquecimento de água por meio da energia solar utilizando materiais descartados é crescente devido ao baixo custo de aquisição, de montagem e da facilidade de instalação e operação (ALANO, 2008; SOUZA et al.,2010; SOCIEDADE DO SOL, 2015).

Estudos realizados com Coletores Solares de Baixo Custo (CSBC) nas condições climáticas de Pelotas-RS, comprovaram o acréscimo significativo da temperatura da água em relaçáo à temperatura ambiente, indicando a viabilidade do uso do sistema para aquecimento da água em residências (FERRAZ e SÁ, 2012; MOREIRA e SÁ, 2017).

Para a correta instalação e funcionamento dos coletores solares é necessária a determinaçáo de parâmetros hidráulicos como vazáo da bomba $\mathrm{e}$ a perda de carga em todos os pontos do sistema (KALOGIROU, 2017).

Dessa forma, objetivou-se quantificar as perdas de carga em coletores solares de baixo custo em relaçáo às características do condutor do fluido (Tubos de PVC e Forro de PVC); ao fluxo do fluido no interior do coletor (paralelo e contínuo) e ao arranjo de montagem dos coletores (em série e em paralelo), quando submetidos à variação de vazáo.

\section{MATERIAL E MÉTODOS}

O estudo foi conduzido no Laboratório de Hidrodinâmica Ambiental do Curso de Tecnologia em Saneamento Ambiental do Instituto Federal Sul-RioGrandense - Campus Pelotas.
Foram analisados três modelos de coletores solares (C1, C2 e C3) construídos a partir da metodologia e de materiais recomendados por ALANO (2008) e SOCIEDADE DO SOL (2015).

O coletor $\mathrm{C} 1$ foi constituído por tubos de PVC soldável de $20 \mathrm{~mm}$ de diâmetro, garrafas PET e embalagens longa vida.

O coletor C2 foi montado com tubos de PVC soldável de $20 \mathrm{~mm}$ de diâmetro e forros de PVC com as dimensôes de 0,2 $\mathrm{m}$ de largura e 0,08 $\mathrm{m}$ de espessura.

$\mathrm{O}$ coletor $\mathrm{C} 3$ foi semelhante ao coletor $\mathrm{C} 2$, diferindo somente em relaçáo às dimensóes do forro de PVC de 0,14 $\mathrm{m}$ de largura e 0,014 $\mathrm{m}$ de espessura. Ambos coletores apresentaram as dimensōes totais de $1,0 \mathrm{~m}$ de largura e 1,0 m de comprimento, correspondendo uma área de 1,0 $\mathrm{m}^{2}$. O coletor de tubos de PVC e forro de PVC, assim como a bancada de testes podem ser observados na Figura 1.

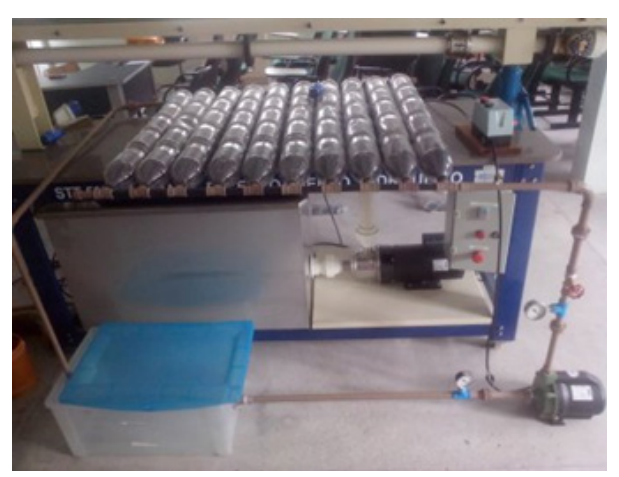

(a)

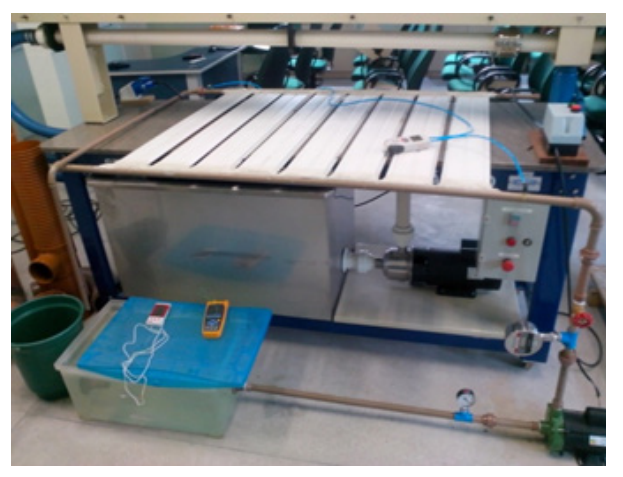

(b)

Figura 1. Instalação do experimento de perda de carga em coletores de tubos de PVC soldável de $20 \mathrm{~mm}$ de diâmetro (a) e coletor de forro de PVC (b)

Os coletores foram instalados em uma bancada de testes onde foi monitorada a perda de carga $(h f)$ por meio de um manômetro diferencial digital de múltipla escala, modelo AK205 com exatidão de $0,01 \mathrm{~cm} \mathrm{H}_{2} \mathrm{O}$ 
e um manômetro de coluna de mercúrio com exatidão de 1,0 mm Hg. As tomadas de pressão foram localizadas na entrada e na saída do fluido nos coletores.

A bancada foi constituída por um reservatório de alimentação com um volume útil de 60 litros e tubos de PVC de $25 \mathrm{~mm}$, alimentado por uma bomba centrífuga monofásica com tensão de $220 \mathrm{~V}$, potência de $1 / 4 \mathrm{CV}$, rotação de $3500 \mathrm{RPM}$, rotor fechado de alumínio com $106 \mathrm{~mm}$ de diâmetro. $\mathrm{Na}$ tubulação de sucção foi instalado um vacuômetro e na tubulaçáo de recalque um manômetro digital para monitoramento da altura manométrica (Hman).

A metodologia utilizada para a determinação da perda de carga ( $h f)$ em CSBC foi baseada na NBR 15747 (ABNT, 2009) e para o cálculo foi utilizada a equação de Bernoulli, descrita na Equação 1.

$\frac{P_{1}}{\gamma}+z_{1}+\frac{V_{1}^{2}}{2 g}=\frac{P_{2}}{\gamma}+z_{2}+\frac{V_{2}^{2}}{2 g}+h f_{1-2}$

Sendo:

$\mathrm{P}_{1}$ - pressão no início do coletor, $\mathrm{m}$;

$\mathrm{P}_{2}$ - pressão no final do coletor, $\mathrm{m}$;

$Z_{1}$ - Posição Vertical do coletor no ponto inicial, m;

$Z_{2}$ - Posição Vertical do coletor no ponto final, m;

$\mathrm{V}_{1}$ - Velocidade do fluido no ponto $1, \mathrm{~m}^{2} \mathrm{~s}^{-1}$;

$\mathrm{V}_{2}$ - Velocidade do fluido no ponto $2, \mathrm{~m}^{2} \mathrm{~s}^{-1}$;

$\mathrm{Y}$ - peso específico do fluido circulante, $\mathrm{kgf} \mathrm{m}^{-3}$;

$\mathrm{g}$ - aceleração da gravidade, $\mathrm{m} \mathrm{s}^{-2}$, e,

$\mathrm{hf}_{1-2}$ - perda de carga, $\mathrm{m}$.

As perdas de carga nos coletores solares foram analisadas nos aspectos: a) condutor do fluido (Tubos de PVC e Forro de PVC); b) fluxo do fluido no interior do coletor (paralelo e contínuo) e c) arranjo dos coletores (em série e em paralelo), em cinco níveis de vazão $\left(0,5 ; 1,0 ; 1,5 ; 2,0\right.$ e $\left.2,5 \mathrm{~m}^{3} \mathrm{~h}^{-1}\right)$.

Os níveis de vazão foram obtidos pela curva característica da bomba, em funçáo da altura manométrica (Hman) e da vazão de bombeamento (Q).

A curva característica Hman $\times Q$ foi obtida pela abertura gradual do registro de gaveta e por registros dos valores instantâneos de vazão, determinado pelo método gravimétrico, e pela leitura da pressão na sucção e no recalque da bomba. Para a determinação da vazão, utilizou-se um recipiente graduado com volume de 10 litros, uma balança digital com precisão de $0,05 \mathrm{~g}$ e um cronômetro digital.

Os resultados obtidos foram submetidos à análise de variância, para o fator qualitativo (coletor) foi realizada a comparação entre médias pelo teste de Tukey à 5\% de significância e para o fator quantitativo (vazão), foi realizada a análise de regressão. Para as análises estatísticas utilizou o software livre $\mathrm{R}$ ( $\mathrm{R}$ CORE TEAM, 2017) e o pacote de dados "ExpDes" (FERREIRA, et al., 2013)

\section{RESULTADOS E DISCUSSÃO}

Nos testes realizados, a bomba centrífuga utilizada operou em uma faixa de vazão de 0 a 2,60 m3 h-1 e forneceu uma altura manométrica de 16,5 a 6,0 metros de coluna de água (mca) respectivamente, atuando dentro dos limites de pressão de serviço de aquecedores solar comerciais.

De acordo com Mesquita et al. (2006), os modelos de simulação costumam-se ajustar à curva característica da bomba centrífuga por meio de um polinômio do segundo grau. Nesse estudo, o ajuste de um polinômio quadrático da relação Hman versus $Q$, $(H \operatorname{man}=0,6076 Q 2-5,5671 Q+16,641)$, representou uma exatidão de $99 \%$ e permitiu ajustar os níveis de vazão utilizados na análise da perda de carga nos coletores solares.

Em relaçáo ao material utilizado como condutor do fluido, verificou-se que ambos os modelos apresentaram um aumento significativo da perda de carga com o incremento da vazáo.

Não foram observadas diferenças significativas entre os coletores para a vazão de $0,5 \mathrm{~m}^{3} \mathrm{~h}^{-1}$. Porém com o aumento da vazáo o coletor de tubo de PVC apresentou a maior perda de energia, diferindo significativamente dos demais coletores (Tabela 1). Já o coletor C3 apresentou a menor perda de carga com o aumento da vazão.

Tabela 1. Perda de carga (mca) observada em função da vazão em coletores constituídos com condutos de PVC (C1), forro de PVC de $8 \mathrm{~mm}$ de espessura (C2) e forro de PVC de $14 \mathrm{~mm}$ de espessura(C3).

\begin{tabular}{|c|c|c|c|c|c|}
\hline \multirow[t]{2}{*}{ Coletor } & \multicolumn{5}{|c|}{ Vazão $\left(\mathrm{m}^{3} \mathrm{~h}^{-1}\right)$} \\
\hline & 0,5 & 1,0 & 1,5 & 2,0 & 2,5 \\
\hline
\end{tabular}




\begin{tabular}{llllll}
\hline $\mathrm{C} 1$ & $0,062 \mathrm{a}$ & $0,27 \mathrm{a}$ & $0,62 \mathrm{a}$ & $1,13 \mathrm{a}$ & $1,42 \mathrm{a}$ \\
$\mathrm{C} 2$ & $0,063 \mathrm{a}$ & $0,13 \mathrm{~b}$ & $0,31 \mathrm{~b}$ & $0,65 \mathrm{~b}$ & $0,96 \mathrm{~b}$ \\
$\mathrm{C} 3$ & $0,079 \mathrm{a}$ & $0,09 \mathrm{~b}$ & $0,26 \mathrm{~b}$ & $0,53 \mathrm{c}$ & $0,81 \mathrm{c}$ \\
\hline
\end{tabular}

Letras minúsculas iguais entre linhas não diferem significativamente pelo teste de Tukey a $5 \%$ de probabilidade.

A perda de energia nos coletores pode estar relacionada com a seçáo do conduto utilizado. Rettore Neto et al. (2013), observaram que a pressão de operaçáo ocasionou o aumento dos diâmetros de tubos de polietileno e, consequentemente, redução da perda de carga contínua nos tubos estudados. Segundo Vilela et al. (2003), alteraçóes nos diâmetros internos, em virtude de variaçóes na pressão de operação, podem ocasionar variaçóes nas perdas de carga superiores a $20 \%$.

Para os valores de vazóes estudados foram observadas perdas de carga de 0,06 a 1,60 mca para o coletor de tubos de PVC (C1), de 0,05 a 1,40 mca para o coletor de forro de PVC de 8,0 $\mathrm{mm}$ de espessura (C2) e de 0,08 a 0,90 mca para o coletor de forro de PVC de 14,0 mm (C3), como pode ser observado na Figura 2.

A variação da perda de carga com o incremento da vazáo nos coletores analisados apresentou um comportamento descrito por um polinominal crescente de $2^{\circ}$ grau, significativa a $1 \%$ de probabilidade.

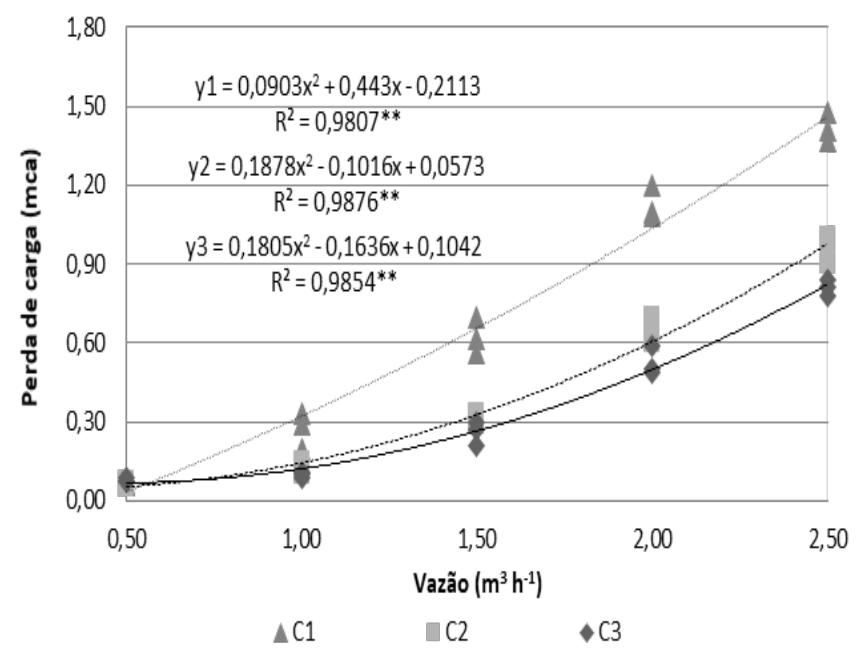

Figura 2. Perda de carga (mca) em coletores solares constituídos com condutos de PVC (C1) e forros de PVC de $8 \mathrm{~mm}$ (C2) e de $14 \mathrm{~mm}$ (C3) de espessura.

Os coletores de placas de PVC apresentaram limitaçóes quanto à pressão de operação, apresentando problemas de vazamento. $\mathrm{O}$ aumento da vazáo ocasionou a ruptura da vedação nos coletores de forro de PVC, o que pode ter influenciado na perda de energia dos mesmos, já que a velocidade de fluxo ao longo do conduto tem influência na variação de energia do fluido (CARVALHO et al., 2008). Por esse motivo os testes de sentido do fluxo e associação dos coletores foram realizados somente com o coletor de PVC de $20 \mathrm{~mm}$ de diâmetro (C1).

Em relação ao sentido do fluxo do fluido no coletor, o coletor de PVC com fluxo contínuo apresentou uma perda de carga significativamente superior em comparaçáo com as perdas verificadas no coletor de fluxo paralelo em todos os valores de vazão analisados, como pode ser observado na Tabela 2 .

Tabela 2. Perda de carga (mca) média em coletores de PVC em relação ao fluxo da água contínuo e paralelo e a instalação dos coletores em série e em paralelo.

\begin{tabular}{cccccc}
\hline \multirow{2}{*}{ Fluxo } & \multicolumn{5}{c}{ Vazão $\left(\mathrm{m}^{3} \mathrm{~h}^{-1}\right)$} \\
\cline { 2 - 6 } & 0,5 & 1,0 & 1,5 & 2,0 & 2,5 \\
\hline Contínuo & $1,53 \mathrm{a}$ & $3,57 \mathrm{a}$ & $6,30 \mathrm{a}$ & $9,46 \mathrm{a}$ & $12,33 \mathrm{a}$ \\
Paralelo & $0,24 \mathrm{~b}$ & $0,52 \mathrm{~b}$ & $1,10 \mathrm{~b}$ & $1,68 \mathrm{~b}$ & $2,05 \mathrm{~b}$ \\
\hline Instalaçáo & \multicolumn{5}{c}{ Vazão $\left(\mathrm{m}^{3} \mathrm{~h}^{-1}\right)$} \\
\cline { 2 - 6 } & 0,5 & 1,0 & 1,5 & 2,0 & 2,5 \\
\hline Série & $0,62 \mathrm{a}$ & $1,93 \mathrm{a}$ & $3,81 \mathrm{a}$ & $5,61 \mathrm{a}$ & $7,08 \mathrm{a}$ \\
Paralelo & $0,13 \mathrm{~b}$ & $0,45 \mathrm{~b}$ & $0,75 \mathrm{~b}$ & $1,35 \mathrm{~b}$ & $1,72 \mathrm{~b}$ \\
\hline
\end{tabular}

Letras minúsculas iguais entre linhas não diferem significativamente pelo teste de Tukey a $5 \%$ de probabilidade.

O aumento da vazão ocasionou um incremento significativo da perda de carga em ambos coletores independente do sentido do fluxo.

No coletor de fluxo contínuo a perda de carga variou polinomialmente, enquanto que a perda de carga no coletor de fluxo paralelo apresentou um comportamento linear significativo em relação aos níveis de vazão (Figura 3).

Isso ocorreu provavelmente devido às características e o número de conexôes utilizadas no modelo de fluxo contínuo que ocasionou o aumento da perda de carga, Oliveira et al. (2011), observaram perdas de carga localizadas em conexóes de PVC crescentes exponencialmente com o aumento da vazáo. 


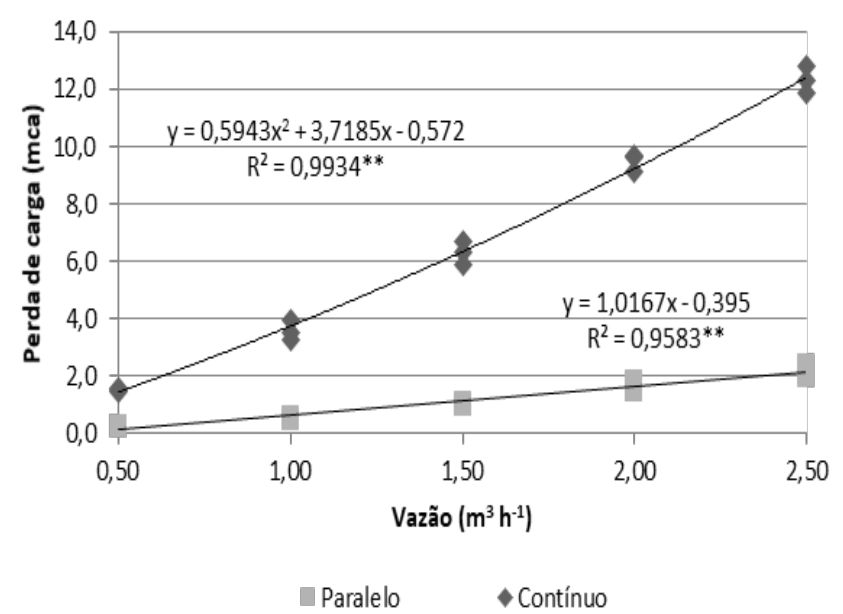

Figura 3. Perda de carga (m) ocasionada pelo incremento da vazão em coletores com fluxo paralelo e fluxo contínuo do fluido.

Em relação à instalação dos coletores, o arranjo em paralelo, ocasionou uma menor perda de carga em comparação ao arranjo em série. A variaçáo da perda de carga nos coletores em paralelo foi em média quatro vezes menor que no arranjo em série, diferindo significativamente entre os arranjos em todos os níveis de vazão (Tabela 2).

A perda de energia piezométrica variou de 0,62 a 7,08 mca nos coletores em série, enquanto nos coletores em paralelo da perda de energia oscilou de 0,13 a 1,72 mca. Em ambos os arranjos a variação da perda de carga apresentou um comportamento linear (Figura 4). Esse comportamento está associado ao incremento da vazão, Prado (2015) verificou que a redução do diâmetro da tubulação e o incremento da vazão promovem um aumento da perda de carga na tubulação.

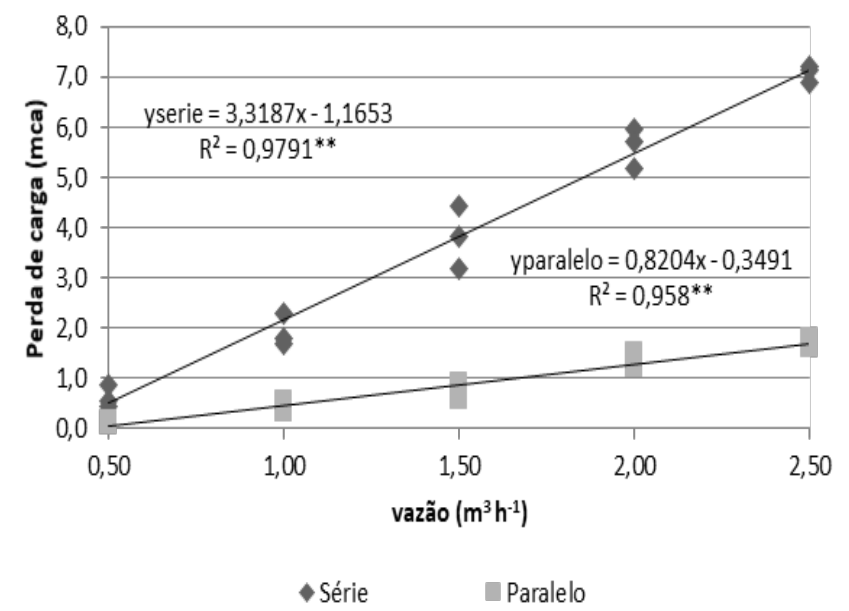

Figura 4. Perda de carga (mca) em função da vazão em relação à instalação em série e em paralelo dos coletores de baixo custo.
Em comparação com valores obtidos em coletores comerciais que apontam uma perda de carga de 0,004 a 0,230 mca (HELIOTEK, 2015), as perdas de energia foram superiores em relação aos CSBC. Esses resultados podem estar relacionados com diâmetro da tubulação utilizado e com vazão de teste adotada. Verificou-se uma relação direta entre a velocidade do fluido e o arranjo dos coletores, existindo relação direta entre as perdas de carga contínua e, principalmente, as perdas de carga localizada em função do aumento de conexóes nos coletores.

\section{CONCLUSÃO}

De acordo com as condiçóes experimentais e conforme os resultados obtidos, pode-se concluir que:

1 - a perda de carga, nos coletores analisados, foi influenciada pela dimensão dos condutos do fluido, pelo sentido do fluxo no interior dos coletores e pelo arranjo de instalação, sendo fatores relevantes no dimensionamento e na instalação de sistemas forçados de aquecimento de água;

2 - o layout construtivo que apresentou resultados positivos (menor perda de carga) foi o coletor constituído de tubos de PVC, instalado em arranjo paralelo, com fluxo de água no sentido paralelo e vazão inferior a $1,0 \mathrm{~m}^{3} \mathrm{~h}^{-1}$.

\section{LITERATURA CITADA}

ALANO, J. A. Aquecedor solar produzido com materiais recicláveis. $4^{a}$ edição SEMA. 2008, 22p. Disponível em < http:// novoportal.celesc.com.br/portal/images/arquivos/manuais/ manual-aquecedor-solar.pdf > Acesso em: 30 abr. 2019.

ASSOCIAÇÃO BRASILEIRA DE NORMAS TÉCNICAS - ABNT. NBR15747/2009. Sistemas solares térmicos e seus componentes - Coletores solares Parte1: Requisitos Gerais. 2009. $11 \mathrm{p}$.

CARVAlHO, J. A.; AQUINO, R. F.; PEREIRA, G. M.; RODRIGUES, R. C.; CAMPOS, T. B. Desempenho de uma bomba centrífuga operando com água residuária do processamento do café. Revista Engenharia Agrícola, Jaboticabal, v.28, p.8694, jan/fev 2008.

FERRAZ, C.; SÁ, J. S. Análise de Comportamento térmico de aquecedores de água de baixo custo. XIII Salâo de Iniciação Científica Pontifícia Universidade Católica do Rio Grande do Sul, Porto Alegre, 2012. 
FERREIRA, E. B.; CAVALCANTI, P. P.; NOGUEIRA, D. A. ExpDes: experimental designs package. $\mathrm{R}$ package version (1.1.2). 2015. Disponível em: <https://cran.r-project.org/web/ packages/ExpDes.pt/ExpDes.pt.pdf>. Acesso: 26 nov. 2016.

GOMES, C. P. Energia Solar: utilização como Fonte de Energia Alternativa. Bolsista de Valor: Revista de divulgação do Projeto Universidade Petrobras e IF Fluminense, v.2, n.1, p.159-163, 2012.

HELIOTEK. Manual do produto coletor solar MC Evolution. Disponível em:<www.heliotek.com.br>. Acesso em: 22 maio 2015.

KALOGIROU, S. Engenharia de Energia Solar: Processos e Sistemas. Elsevier Brasil, p.864, 2017.

LOPO, A. B. Análise do Desempenho Térmico de um sistema de Aquecimento Solar de Baixo Custo Dissertação (Mestrado em Engenharia Mecânica) - Universidade Federal de Minas Gerais, Fevereiro de 2010.

MESQUITA, A. L. A.; SILVA, L. J. S.; ABRAHIM JÚNIOR, O.; PEREIRA, J. A. R.; SILVA, A. C. P. Aspectos importantes na utilização de bombas centrífugas em velocidade variável para a redução do consumo de energia. Seminário de Abastecimento Urbano de Água. Anais João Pessoa: Universidade Federal Paraíba, 2006.

MOREIRA, G. C.; SÁ, J. S. Análise do desempenho de materiais recicláveis no aquecimento de água por meio da energia solar. Revista Gestáo \& Sustentabilidade Ambiental, Florianópolis, v. 6, n. 1, p.41- 66, abr./set, 2017.

OLIVEIRA, F. G.; RESENDE, P. R. N.; CRUZ, O. C.; BARRETO, A. C.; SERAPHIM, O. J. Perda de carga em reduçóes concêntricas de PVC. Global Science Technology, Goiás, v. 04, n. 02 , p. 83 - 90, mai/ago. 2011.

PRADO, G. Decréscimo da energia de pressão devido a instalação de conexôes e peças especiais nas tubulaçôes. Enciclopédia Biosfera, Centro Científico Conhecer - Goiânia, v.11 n.21; p. 2015.

R CORE TEAM. R. The R Project for Statistical Computing. Disponível em: <http://www.r-project.org/>. Acesso em 15 abril 2017.

RETTORE NETO, O.; BOTREL, T. A.; FRIZZONE, J. A.; PINTO, M. F.; CAMARGO, A. P. Quantificação do erro na determinação da perda contínua de carga em tubos elásticos. Engenharia Agrícola, Jaboticabal, v.33, n.6, p.1312-1321, nov./ dez. 2013.

SILVA, R. G; CARMO, M. J. Energia solar fotovoltaica: uma proposta para melhoria da gestáo energética International Scientific Journal. n.2, v. 12, p. 129-173, 2017.

SOCIEDADE DO SOL. Aquecedor Solar de Baixo Custo. Disponível em: <http://www.sociedadedosol.org.br/>Acesso em 23 abril 2015.
SOUZA, L. G. M.; LOPO, A. B., MENDES, U. L.; DE SOUZA, M. C. S., SANTOS, N. R. G.; DE SOUZA, L. G. V. M. Coletor Solar Aletado com Tubos de Policloreto de Vinila formando um Novo Modelo de Configuração em Série, VI Congresso Nacional de Engenharia Mecânica, Campina Grande/PB,2010.

VILELA, L. A. A.; SOCCOL, O. J.; GERVÁSIO, E. S.; FRIZZONE, J. A.; BOTREL, T. A. Alteração no diâmetro e na perda de carga em tubos de polietileno submetidos a diferentes pressóes. Revista Brasileira de Engenharia Agrícola e Ambiental, Viçosa, v.7, n.1, p.182-185, 2003. 